\title{
A Note on the Transience of Critical Branching Random Walks on the Line
}

\author{
Gerold Alsmeyer and Matthias Meiners \\ Institut für Mathematische Statistik, Fachbereich Mathematik, Einsteinstraße 62, D-48149 Münster, Germany
}

\begin{abstract}
Gantert and Müller (2006) proved that a critical branching random walk (BRW) on the integer lattice is transient by analyzing this problem within the more general framework of branching Markov chains and making use of Lyapunov functions. The main purpose of this note is to show how the same result can be derived quite elegantly and even extended to the nonlattice case within the theory of weighted branching processes. This is done by an analysis of certain associated random weighted location measures which, upon taking expectations, provide a useful connection to the well established theory of ordinary random walks with i.i.d. increments. A brief discussion of the asymptotic behavior of the left- and rightmost particles in a critical BRW as time goes to infinity is provided in the final section by drawing on recent work by $\mathrm{Hu}$ and Shi (2008).
\end{abstract}

Keywords: branching random walk, critical regime, recurrence, transience, minimal and maximal position, random weighted location measure, renewal theory

\section{Introduction}

Consider a cloud of particles which moves on the line as follows. Initially there is one particle sitting at the origin which after one unit of time splits into a random number of new particles having distribution $\left(p_{j}\right)_{j \geq 0}$, where $p_{0}=0$. The daughter particles are then independently displaced relative to their mother's site in accordance with the same step size distribution $Q$, say. This process continues indefinitely, i.e., each new born particle splits after one unit of time in accordance with $\left(p_{j}\right)_{j \geq 0}$, and the relative displacement of each daughter particle with respect to its mother's site has distribution $Q$ and is independent of the relative displacements of its siblings as well as of the history of the process. This model describes a special nonextinctive BRW, the specialization being that the relative displacements of siblings (given their total number) are i.i.d. rather than chosen from a general point process on $\mathbb{R}$. Likewise, one may adopt the viewpoint as in Gantert and Müller (2006) that any new born particle lives forever and performs a random walk with step size distribution $Q$. Right before each jump it produces $j-1$ daughter particles with probability $p_{j}(j \geq 1)$ which start independent random walks of the same kind at initial positions relative to their mother's site chosen in accordance with $Q$. Note that the cloud size evolves as a simple nonextinctive Galton-Watson process $\left(Z_{n}\right)_{n \geq 0}$ with one ancestor and offspring distribution $\left(p_{j}\right)_{j \geq 0}$. A more detailed specification of the model will be given in Section 2 after having described the main problem to be addressed in this note.

1365-8050 @ 2008 Discrete Mathematics and Theoretical Computer Science (DMTCS), Nancy, France 
Suppose that $Q$ has positive mean but also positive mass on $(-\infty, 0)$. Then all particles in the cloud are moving towards $\infty$ with probability one while, on the other hand, their trajectories will also have negative excursions. Hence the ever increasing number of independently moving particles might cause bounded neighborhoods of 0 be visited infinitely often if the cloud is growing fast enough. We are thus led to the question whether there exists a branching threshold $m^{*}$ such that any bounded neighborhood of 0 (or any other $x \in \mathbb{R}$ in the same irreducibility class) is recurrent, i.e., a.s. visited by infinitely many particles, if $m>m^{*}$, while being transient, if $m<m^{*}$, where $m:=\sum_{j \geq 1} j p_{j}$ is the mean offspring (including the reproducing particle). In the case of an irreducible lattice random walk, the positive answer has been given by Comets et al. (1998) in their analysis of the more general BRW in random environment. Moreover, transience holds true in the boundary case $m=m^{*}$, as recently been proved by Gantert and Müller (2006) within the more general framework of branching Markov chains where the random walk is replaced with an arbitrary irreducible Markov chain on a countable state space, see Benjamini and Peres (1994) for the basics and also the classification of possible regimes of such models as to their recurrence behavior. Further results on the recurrence or transience of various generalizations of the classical BRW may be found in Machado and Popov (2000, 2003); Machado et al. (2001); Menshikov and Volkov (1997). An essential tool in these works is the use of Lyapunov test functions, and this constitutes the main difference to the present note. Our main purpose is in fact to demonstrate how certain random weighted location measures to be defined in Section 2 and their connection to renewal and fluctuation theory for classical random walks (cf. Section 3) may be utilized as an alternative tool in order to not only reproduce the afore-mentioned results for lattice BRWs but to provide also without much additional effort an extension to the situation where $Q$ is nonlattice. Our main result will be stated in Section 2 after the necessary formal details including a definition of recurrence and transience for BRWs. The proof will be given in Section 4. Finally, Section 5 provides some fairly sharp information on the position of the leftmost and the rightmost particle in a critical BRW as time goes to infinity. Our theorems stated there follow without much ado from a recent result by $\mathrm{Hu}$ and Shi (2008).

\section{Model description and main results}

Let $\mathbb{V}$ be the infinite Ulam-Harris tree with vertex set $\bigcup_{n \geq 0} \mathbb{N}^{n}$ where $\mathbb{N}=\{1,2, \ldots\}$ denotes the set of positive integers and $\mathbb{N}^{0}:=\{\varnothing\}$ by convention. Each vertex $v=\left(v_{1}, \ldots, v_{n}\right)$ of length $|v|=n$, shortly written as $v_{1} v_{2} \ldots v_{n}$ hereafter, is uniquely connected to the root $\varnothing$ by the path $\varnothing \rightarrow v_{1} \rightarrow v_{1} v_{2} \rightarrow$ $\ldots \rightarrow v_{1} v_{2} \ldots v_{n}$. If $w=w_{1} \ldots w_{m}$ denotes another vertex, we write $v w$ for the concatenation of $v$ and $w$, i.e., for $v_{1} \ldots v_{n} w_{1} \ldots w_{m}$. In the present context, each $v$ is interpreted as a (potential) particle of the $n$-th generation. It is the mother of the successors $v i:=v_{1} \ldots v_{n} i, i \in \mathbb{N}$, and an ancestor of any $v w, w \in \mathbb{V}$. In places where it occurs $v_{1} \ldots v_{n}:=\varnothing$ is stipulated whenever $n=0$.

The following weighted branching model assigns a random weight $L(v) \in\{0,1\}$ and a random position $S(v)$ in $\mathbb{R}$ to each node $v$ of the tree, where $L(v)=1$ means that particle $v$ is actually alive. Let $(\Omega, \mathfrak{A}, \mathbb{P})$ be a given probability space which carries i.i.d. random sequences

$$
T(v) \otimes X(v):=\left(T_{i}(v), X_{i}(v)\right)_{i \geq 1}: \Omega \rightarrow(\{0,1\} \times \mathbb{R})^{\mathbb{N}}, \quad v \in \mathbb{V}
$$

Furthermore, $\left(T_{i}(v)\right)_{i \geq 1}$ and $\left(X_{i}(v)\right)_{i \geq 1}$ are independent as well for each $v \in \mathbb{V}$ with

$$
\mathbb{P}\left(T_{1}(v)=\ldots=T_{j}(v)=1, T_{j+1}(v)=T_{j+2}(v)=\ldots=0\right)=p_{j}
$$


for $j \geq 1$ and $X_{1}(v), X_{2}(v), \ldots$ being i.i.d. having common distribution $Q$. Put $L(\varnothing):=1, S(\varnothing):=0$, and define recursively

$$
L(v i):=L(v) T_{i}(v) \text { and } S(v i):=S(v)+X_{i}(v)
$$

for $v=v_{1} \ldots v_{n} \in \mathbb{V}$ and $i \in \mathbb{N}$, thus

$$
L(v)=\prod_{j=1}^{n} T_{v_{j}}\left(v_{1} \ldots v_{j-1}\right) \quad \text { and } \quad S(v)=\sum_{j=1}^{n} X_{v_{j}}\left(v_{1} \ldots v_{j-1}\right) .
$$

The total size of the $n$-th generation (number of particles alive at time $n$ ) is now given by

$$
Z_{n}:=\sum_{|v|=n} L(v)
$$

for $n \geq 0$ and forms a simple Galton-Watson process with offspring distribution $\left(p_{j}\right)_{j \geq 0}$. Throughout this article, we will make the standing assumption that $\left(Z_{n}\right)_{n \geq 0}$ is supercritical, i.e., $m>1$ or, equivalently, $p_{1}<1$ (as $\left.p_{0}=0\right)$. In order to describe the positions of all living particles at time $n$, we introduce the random location measures

$$
\Pi_{n}:=\sum_{|v|=n} L(v) \delta_{S(v)}, \quad n \geq 0
$$

and call $\left(\Pi_{n}\right)_{n \geq 0}$ a BRW on $\mathbb{R}$ with offspring distribution $\left(p_{j}\right)_{j \geq 0}$ and increment distribution $Q$. Put also

$$
\Pi:=\sum_{n \geq 0} \Pi_{n}=\sum_{v \in \mathbb{V}} L(v) \delta_{S(v)}
$$

which is the associated overall empirical occupation measure and will be called branching renewal measure of $\left(\Pi_{n}\right)_{n \geq 0}$.

Definition 2.1 A BRW $\left(\Pi_{n}\right)_{n \geq 0}$ with increment distribution $Q$ is called $d$-arithmetic if $Q$ is $d$-arithmetic, i.e., if

$$
d:=\sup \{c>0: Q(c \mathbb{Z})=1\}>0,
$$

and is called nonarithmetic ( 0 -arithmetic) otherwise.

So the lattice-span of $\left(\Pi_{n}\right)_{n \geq 0}$ is just the lattice-span $d$ of its increment distribution $Q(=0$ in the nonarithmetic case). Notice that $d=\infty$ if $Q=\delta_{0}$. Excluding this case, we may and will assume hereafter w.l.o.g. that $d=1$ whenever $d>0$. It is convenient to further define $\mathbb{G}_{0}:=\mathbb{R}$ and $\mathbb{G}_{1}:=\mathbb{Z}$.

Since our interest lies in those BRWs that do not move in one direction only we make the standing assumption hereafter that the increment distribution $Q$ puts mass on $(-\infty, 0)$ as well as $(0, \infty)$, that is,

$$
Q((-\infty, 0)) \wedge Q((0, \infty))>0 .
$$

Such a $Q$ as well as an associated BRW is called genuinely two-sided hereafter. The definitions of recurrence and transience for a genuinely two-sided BRW $\left(\Pi_{n}\right)_{n \geq 0}$ are preceded by the following classification result (a zero-one law) for its branching renewal measure $\Pi$. For intervals $I \subset \mathbb{R}$, let $H_{I}: \mathbb{R} \rightarrow[0,1]$ be the function given by

$$
H_{I}(t):=\mathbb{P}(\Pi(t+I)<\infty), \quad t \in \mathbb{R} .
$$

Then put $H:=H_{(-\infty, 0)}$, thus $H(t)=\mathbb{P}(\Pi((-\infty, t))<\infty)$, and $H_{\varepsilon}:=H_{(-\varepsilon, \varepsilon)}$, thus $H_{\varepsilon}(t)=$ $\mathbb{P}(\Pi((t-\varepsilon, t+\varepsilon))<\infty)$. 
Proposition 2.2 Let $\left(\Pi_{n}\right)_{n \geq 0}$ be a genuinely two-sided, d-arithmetic $B R W, d \in\{0,1\}$, with increment distribution $Q$. Suppose also $p_{0}=0$ and $p_{1}<1$. Then either $H_{\varepsilon} \equiv 0$ for all $\varepsilon>0$, or $H_{\varepsilon} \equiv 1$ for all $\varepsilon>0$. Similarly, either $H \equiv 0$ or $H \equiv 1$.

Remark 2.3 (a) Plainly, in the 1-arithmetic case the dichotomy for the $H_{\varepsilon}$ reduces to the statement that the $\Pi(\{n\}), n \in \mathbb{Z}$, are either all a.s. finite or all a.s. infinite. Moreover, a reflection argument (replace $X(v)$ with $-X(v)$ for each $v \in \mathbb{V})$ shows that the zero-one dichotomy holds for $H_{(0, \infty)}$ as well.

(b) If $Q$ is concentrated on one halfline and having an atom at 0 , then Proposition 2.2 may fail. For instance, if $Q\left(\mathbb{N}_{0}\right)=1, p:=Q(\{0\}) \in(0,1)$ and $m p>1$, then it is easily seen that $\mathbb{P}(\Pi(\{0\})<\infty)$ $\left(=H_{\varepsilon}(0)\right.$ for $\left.\varepsilon \in(0,1)\right)$ equals the extinction probability $q^{*} \in(0,1)$ of the supercritical Galton-Watson process defined as $Z_{n}^{*}:=\sum_{|v|=n} L(v) \mathbf{1}_{\{S(v)=0\}}, n \geq 0$. The function $H$ in this situation equals 1 on $(-\infty, 0]$, takes values in $\left(0, q^{*}\right]$ on $(0, \infty)$ and converges to 0 as $t \rightarrow \infty$.

Definition 2.4 (a) A genuinely two-sided 1-arithmetic BRW $\left(\Pi_{n}\right)_{n \geq 0}$ is called recurrent if

$$
\Pi(\{k\})=\sum_{n \geq 0} \Pi_{n}(\{k\})=\infty \text { a.s. }
$$

for some (and then all) $k \in \mathbb{Z}$, and transient otherwise.

(b) A genuinely two-sided nonarithmetic BRW $\left(\Pi_{n}\right)_{n \geq 0}$ is called (topologically) recurrent if

$$
\Pi(I)=\sum_{n \geq 0} \Pi_{n}(I)=\infty \text { a.s. }
$$

for some (and then all) nonempty bounded open intervals $I$, and transient otherwise.

In order to present our main result, we now confine to the case where $Q$ has finite positive mean $\mu(Q)$. Defining the Laplace transform of $Q$

$$
\Psi(\theta):=\int e^{-\theta x} Q(d x)
$$

with domain $\mathbb{D}_{\Psi}:=\{\theta: \Psi(\theta)<\infty\}$, we make the additional assumption that there exists a (necessarily unique) $\vartheta>0$ such that

$$
\int|x| e^{-\vartheta x} Q(d x)<\infty \quad \text { and } \quad \Psi^{\prime}(\vartheta)=-\int x e^{-\vartheta x} Q(d x)=0 .
$$

Positivity of $\vartheta$ follows from $\Psi^{\prime}(0)=-\mu(Q)<0$ and the convexity of $\Psi$ on $\mathbb{D}_{\Psi}$ (which contains at least $[0, \vartheta]$ ). If $\vartheta$ is not an interior point of $\mathbb{D}_{\Psi}$, then $\Psi^{\prime}(\vartheta)$ is actually the left-hand derivative of $\Psi$ at $\vartheta$

Theorem 2.5 Let $\left(\Pi_{n}\right)_{n \geq 0}$ be a genuinely two-sided, d-arithmetic BRW, $d \in\{0,1\}$, with increment distribution $Q$. Suppose also $p_{0}=0, p_{1}<1, \mu(Q) \in(0, \infty)$, and that (3) holds true. Then $\left(\Pi_{n}\right)_{n \geq 0}$ is recurrent, if $m \Psi(\vartheta)>1$, and transient otherwise.

In view of this result, the BRW $\left(\Pi_{n}\right)_{n \geq 0}$ is called critical, if $m \Psi(\vartheta)=1$, subcritical, if $m \Psi(\vartheta)<1$, and supercritical, if $m \Psi(\vartheta)>1$. 


\section{Random weighted location measures and an associated ran- dom walk}

This section is devoted to the necessary prerequisites in order to prove our main results in the next section. We start by defining the random weighted location measures (r.w.l.m.)

$$
\Lambda_{n}:=m^{-n} \Pi_{n}=m^{-n} \sum_{|v|=n} L(v) \delta_{S(v)}, \quad n \geq 0
$$

as well as their multivariate extensions

$$
\Lambda_{0: n}:=m^{-n} \sum_{|v|=n} L(v) \delta_{\mathbf{S}(v)}, \quad n \geq 0
$$

where

$$
\mathbf{S}(v):=\left(S_{0}(v), S_{1}(v), \ldots, S_{n-1}(v), S_{n}(v)\right),
$$

with $S_{k}(v):=S\left(v_{1} \ldots v_{k}\right)$ if $v=v_{1} \ldots v_{n}$ and $0 \leq k \leq n$. Since $\Lambda_{0: n}\left(\mathbb{R}^{n+1}\right)=\Lambda_{n}(\mathbb{R})=m^{-n} Z_{n}, n \geq 0$, forms a positive martingale with mean one, we see that

$$
\bar{\Lambda}_{n}:=\mathbb{E} \Lambda_{n} \quad \text { and } \quad \bar{\Lambda}_{0: n}:=\mathbb{E} \Lambda_{0: n}
$$

are both probability distributions for each $n$. Of course, $\mathbb{E} \Lambda_{n}$ and $\mathbb{E} \Lambda_{0: n}$ are the measures more explicitly given by

$$
\left(\mathbb{E} \Lambda_{n}\right)(A)=\mathbb{E} \Lambda_{n}(A) \quad \text { and } \quad\left(\mathbb{E} \Lambda_{0: n}\right)(B)=\mathbb{E} \Lambda_{0: n}(B)
$$

for measurable $A \subset \mathbb{R}$ and $B \subset \mathbb{R}^{n+1}$.

For any $\theta \in \mathbb{D}_{\Psi}$, let us further define the r.w.l.m.

$$
\begin{aligned}
\Lambda_{n}^{\theta} & :=\frac{1}{(m \Psi(\theta))^{n}} \sum_{|v|=n} L(v) e^{-\theta S(v)} \delta_{S(v)}, \quad \bar{\Lambda}_{n}^{\theta}:=\mathbb{E} \Lambda_{n}^{\theta}, \\
\Lambda_{0: n}^{\theta} & :=\frac{1}{(m \Psi(\theta))^{n}} \sum_{|v|=n} L(v) e^{-\theta S(v)} \delta_{\mathbf{S}(v)}, \quad \bar{\Lambda}_{0: n}^{\theta}:=\mathbb{E} \Lambda_{0: n}^{\theta}
\end{aligned}
$$

as well as the probability distribution

$$
Q_{\theta}(d x):=\Psi(\theta)^{-1} e^{-\theta x} Q(d x) .
$$

We point out that the $\Lambda_{n}^{\theta}$ and $\Lambda_{0: n}^{\theta}$ are the counterparts of $\Lambda_{n}$, respectively $\Lambda_{0: n}$ for the weighted branching

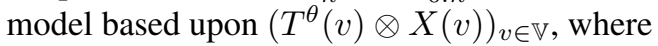

$$
T^{\theta}(v):=\left(\Psi(\theta)^{-1} e^{-\theta X_{i}(v)} T_{i}(v)\right)_{i \geq 1} .
$$

Plainly, $\Lambda_{n}=\Lambda_{n}^{0}, \Lambda_{0: n}=\Lambda_{0: n}^{0}, T(v)=T^{0}(v)$ and $Q=Q_{0}$.

The following two lemmata provide the connection to random walks. The first of them has been given in various places, see Lemma 4.1 in Biggins and Kyprianou (1997), Proposition 11 in Biggins and Kyprianou (2005), Lemma 1 in Bingham and Doney (1975), or p. 289 in Durrett and Liggett (1983). 
Lemma 3.1 For each $\theta \in \mathbb{D}_{\Psi}$, let $\mathbb{P}_{\theta}(=\mathbb{P}$ if $\theta=0)$ be a probability measure on $(\Omega, \mathfrak{A})$ and $\left(\bar{S}_{n}\right)_{n \geq 0}$ a sequence of random variables on $(\Omega, \mathfrak{A})$ which, under $\mathbb{P}_{\theta}$, constitutes a random walk with $\bar{S}_{0}:=0$ and increment distribution $Q_{\theta}$. Then

$$
\bar{\Lambda}_{0: n}^{\theta}=\mathbb{P}_{\theta}\left(\left(\bar{S}_{0}, \ldots, \bar{S}_{n}\right) \in \cdot\right),
$$

in particular $\bar{\Lambda}_{n}^{\theta}=Q_{\theta}^{* n}$ for all $n \geq 0$.

We will also need an extension of the previous lemma to a certain class of stopping lines, called homogeneous stopping lines $(H S L)$ hereafter. Let $\underline{\sigma}: \mathbb{R}^{\mathbb{N}_{0}} \rightarrow \mathbb{N}_{0} \cup\{\infty\}$,

$$
\underline{\sigma}\left(s_{0}, s_{1}, \ldots\right):=\inf \left\{n \geq 0:\left(s_{0}, \ldots, s_{n}\right) \in B_{n}\right\}
$$

be any formal stopping rule where $B_{n} \in \mathfrak{B}\left(\mathbb{R}^{n+1}\right)$ for $n \geq 0$ and $\inf \emptyset:=\infty$, and let

$$
\Upsilon_{n}:=\pi_{0: n}(\{\underline{\sigma}=n\}),
$$

for $n \geq 0$ where $\pi_{0: n}$ denotes the projection $\left(s_{k}\right)_{k \geq 0} \mapsto\left(s_{0}, \ldots, s_{n}\right)$. For $\mathbf{v}=\left(v_{1}, v_{2}, \ldots\right) \in \mathbb{N}^{\mathbb{N}}$ (viewed as the boundary of $\mathbb{V}$ ), we further define

$$
\sigma_{\mathbf{v}}:=\underline{\sigma}(\mathbf{S}(\mathbf{v})), \quad \mathbf{S}(\mathbf{v})=\left(S_{n}(\mathbf{v})\right)_{n \geq 0}:=\left(S(\varnothing), S\left(v_{1}\right), S\left(v_{1} v_{2}\right), \ldots\right),
$$

and then

$$
\mathcal{S}:=\left\{\mathbf{v} \mid \sigma_{\mathbf{v}}: \mathbf{v} \in \mathbb{N}^{\mathbb{N}}\right\} \cap \mathbb{V}=\left\{\mathbf{v} \mid \sigma_{\mathbf{v}}: \mathbf{v} \in \mathbb{N}^{\mathbb{N}}, \sigma_{\mathbf{v}}<\infty\right\}
$$

where $\mathbf{v}|0:=\varnothing, \mathbf{v}| n:=v_{1} \ldots v_{n}$ for $n \in \mathbb{N}$, and $\mathbf{v} \mid \infty:=\mathbf{v}$. We call $\mathcal{S}$ the HSL associated with $\underline{\sigma}$. It consists of all nodes $v \in \mathbb{V}$ that are obtained as stopping places when applying the same rule $\underline{\sigma}$ to the random walks $\mathbf{S}(\mathbf{v})$ along all infinite paths $\mathbf{v}$ of the tree. Notice that $\mathcal{S}$ may be empty and that $\mathcal{S}=\{v:|v|=n\}$ in the case $\underline{\sigma} \equiv n$. Stopping lines, also called optional lines, have been defined in varying generality in the literature, the most general one appearing in Jagers (1989), which also provides the basic framework. We mention further Chauvin (1991), Kyprianou (2000), and Biggins and Kyprianou (2004) and note that the last reference contains the definition that is closest to that of an HSL and called very simple line there.

Lemma 3.2 Given any $H S L \mathcal{S}$ associated with a stopping rule $\underline{\sigma}$, put $\sigma:=\underline{\sigma}\left(\bar{S}_{0}, \bar{S}_{1}, \ldots\right)$. Then the following assertions hold true for each $\theta \in \mathbb{D}_{\Psi}$ :

$$
\begin{aligned}
& \mathbb{P}_{\theta}\left(\left(\bar{S}_{0}, \ldots, \bar{S}_{n}\right) \in B, \sigma=n\right) \\
& \quad=\frac{1}{(m \Psi(\theta))^{n}} \mathbb{E}\left(\sum_{v \in \mathcal{S},|v|=n} L(v) e^{-\theta S(v)} \delta_{\mathbf{S}(v)}(B)\right)=\bar{\Lambda}_{0: n}^{\theta}\left(B \cap \Upsilon_{n}\right)
\end{aligned}
$$

for all $n \geq 0$ and $B \in \mathfrak{B}\left(\mathbb{R}^{n+1}\right)$, in particular

$$
\mathbb{P}_{\theta}(\sigma=n)=\frac{1}{(m \Psi(\theta))^{n}} \mathbb{E}\left(\sum_{v \in \mathcal{S},|v|=n} L(v) e^{-\theta S(v)}\right)=\bar{\Lambda}_{0: n}^{\theta}\left(\Upsilon_{n}\right)
$$


for each $n \geq 0$ and

$$
\mathbb{P}_{\theta}(\sigma<\infty)=\sum_{n \geq 0} \bar{\Lambda}_{0: n}^{\theta}\left(\Upsilon_{n}\right)=\mathbb{E}\left(\sum_{n \geq 0} \Lambda_{0: n}^{\theta}\left(\Upsilon_{n}\right)\right)
$$

Finally, putting $Z_{\mathcal{S}}^{\theta}:=\sum_{v \in \mathcal{S}} L(v) e^{-\theta S(v)}$ and $Z_{\mathcal{S}}:=Z_{\mathcal{S}}^{0}=\sum_{v \in \mathcal{S}} L(v)$,

$$
\mathbb{E} Z_{\mathcal{S}}^{\theta}=\mathbb{E}_{\theta}(m \Psi(\theta))^{\sigma} \mathbf{1}_{\{\sigma<\infty\}} \quad \text { and } \quad \mathbb{E} Z_{\mathcal{S}}=\mathbb{E} m^{\sigma} \mathbf{1}_{\{\sigma<\infty\}}
$$

for all $\theta \in \mathbb{D}_{\Psi}$.

Proof: Noting $\{\sigma=n\}=\left\{\left(\bar{S}_{0}, \ldots, \bar{S}_{n}\right) \in \Upsilon_{n}\right\}$ and the fact that $v \in \mathcal{S},|v|=n$ holds iff $\mathbf{S}(v) \in \Upsilon_{n}$, we infer from Lemma 3.1

$$
\begin{aligned}
\mathbb{P}_{\theta}\left(\left(\bar{S}_{0}, \ldots, \bar{S}_{n}\right) \in B, \sigma=n\right) \\
=\mathbb{P}_{\theta}\left(\left(\bar{S}_{0}, \ldots, \bar{S}_{n}\right) \in B \cap \Upsilon_{n}\right) \\
=\bar{\Lambda}_{n}^{\theta}\left(B \cap \Upsilon_{n}\right) \\
=\frac{1}{(m \Psi(\theta))^{n}} \mathbb{E}\left(\sum_{|v|=n} L(v) e^{-\theta S(v)} \delta_{\mathbf{S}(v)}\left(B \cap \Upsilon_{n}\right)\right) \\
=\frac{1}{(m \Psi(\theta))^{n}} \mathbb{E}\left(\sum_{v \in \mathcal{S},|v|=n} L(v) e^{-\theta S(v)} \delta_{\mathbf{S}(v)}(B)\right)
\end{aligned}
$$

which proves (4). The assertions (5) and (6) being direct consequences, let us directly turn to (7). But for $\theta \in \mathbb{D}_{\Psi}$, we infer with the help of [5]

$$
\begin{aligned}
\mathbb{E} Z_{\mathcal{S}}^{\theta} & =\sum_{n \geq 0} \mathbb{E}\left(\sum_{v \in \mathcal{S},|v|=n} L(v) e^{-\theta S(v)}\right) \\
& =\sum_{n \geq 0}(m \Psi(\theta))^{n} \mathbb{P}_{\theta}(\sigma=n) \\
& =\mathbb{E}_{\theta}(m \Psi(\theta))^{\sigma} \mathbf{1}_{\{\sigma<\infty\}}
\end{aligned}
$$

and thus the first half of (7). For the second choose $\theta=0$.

In view of the previous result a HSL $\mathcal{S}$ associated with $\underline{\sigma}$ will be called hereafter HSL associated with $\sigma$, where $\sigma=\underline{\sigma}\left(\bar{S}_{0}, \bar{S}_{1}, \ldots\right)$.

\section{Proofs of Proposition 2.2 and Theorem 2.5}

The following common partial order relations $\prec$ and $\preceq$ on $\mathbb{V}$ will be needed hereafter: Write $v \prec w$ if $v \neq w$ and $v$ belongs to the ancestral line of $w$, while $v \preceq w$ also allows $v=w$. Moreover, $v \prec(\preceq) C$ 
for any $C \subset \mathbb{V}$ shall mean that $w \npreceq(\nprec) v$ for all $w \in C$. Now we can define the pre-S random location measure as

$$
\Pi_{\S}^{\prec}:=\sum_{v \prec \mathcal{S}} L(v) \delta_{S(v)}
$$

for an arbitrary HSL $\mathcal{S}$. Finally, put also

$$
\mathbb{T}:=\{v \in \mathbb{V}: L(v)=1\} \quad \text { and } \quad \mathbb{T}_{n}:=\{v \in \mathbb{T}:|v|=n\} .
$$

The following lemma provides the key to the proof of Proposition 2.2 .

Lemma 4.1 In the situation of Proposition 2.2 the function $H_{I}$ is constant for each nonempty open interval $I$.

Proof: Note that $H_{I}(t)=H_{t+I}(0)$ for all intervals $I$ and $t \in \mathbb{R}$. Hence it suffices to verify that, fixing any nonempty open $I$ with $H_{I}(0)<1$ and any $t \in \mathbb{G}_{d}$, we have $H_{I}(t)=H_{I}(0)$.

Case 1. $I$ bounded.

Since $Q$ is genuinely two-sided, the associated random walk $\left(\bar{S}_{n}\right)_{n \geq 0}$ is topologically irreducible on $\mathbb{G}_{d}$, that is,

$$
\sup _{n \geq 1} \mathbb{P}\left(\bar{S}_{n} \in(x-\varepsilon, x+\varepsilon)\right)>0
$$

for all $x \in \mathbb{G}_{d}$ and $\varepsilon>0$. For the subsequent argument, we restrict ourselves to the nonarithmetic case, the arithmetic one being even simpler. If $I=(x, x+4 \varepsilon)$ for some $x \in \mathbb{R}$ and $\varepsilon>0$, put $I_{1}:=(x, x+2 \varepsilon), I_{2}:=(x+\varepsilon, x+3 \varepsilon)$, and $I_{3}:=(x+2 \varepsilon, x+4 \varepsilon)$. By [8], there are $k_{1}, k_{2}, k_{3}$, such that

$$
\begin{aligned}
& \mathbb{P}\left(\bar{S}_{k_{1}} \in(t, t+\varepsilon)\right)>0, \\
& \mathbb{P}\left(\bar{S}_{k_{2}} \in(t-\varepsilon, t)\right)>0, \\
\text { and } \quad & \mathbb{P}\left(\bar{S}_{k_{3}} \in(t-2 \varepsilon, t-\varepsilon)\right)>0 .
\end{aligned}
$$

Consequently, with $k:=k_{1} \vee k_{2} \vee k_{3}$,

$$
\beta:=\inf _{s \in I} \mathbb{P}\left(\bar{S}_{n+j} \in t+I \text { for some } 1 \leq j \leq k \mid \bar{S}_{n}=s\right)>0 .
$$

Next, since $H_{I}(0)<1$, the event $\{\Pi(I)=\infty\}$ has positive probability $1-H_{I}(0)$, and on this event the stopping times $\sigma_{0}:=0$,

$$
\sigma_{i}:=\inf \left\{n>\sigma_{i-1}+k: S(v) \in I \text { for some } v \in \mathbb{T}_{n}\right\}, \quad i \geq 1,
$$

are all a.s. finite. Let $v^{i}$ be the leftmost (with respect to lexicographic ordering) vertex in $\mathbb{T}_{\sigma_{i}}$ such that $S\left(v^{i}\right) \in I(i \geq 1)$. Then it follows with the help of the strong Markov property that

$$
\begin{aligned}
\mathbb{P}( & \left.\Pi(I)=\infty, S\left(v^{i} \mathbf{1}_{j}\right) \notin t+I \text { for } 1 \leq i \leq n \text { and } 1 \leq j \leq k\right) \\
\leq & \mathbb{P}\left(\sigma_{n}<\infty, S\left(v^{i} \mathbf{1}_{j}\right) \notin t+I \text { for } 1 \leq i \leq n \text { and } 1 \leq j \leq k\right) \\
= & \int_{I} \mathbb{P}\left(\bar{S}_{j} \notin t+I \text { for } 1 \leq j \leq k \mid \bar{S}_{0}=s\right) \\
& \quad \times \mathbb{P}\left(S\left(v^{n}\right) \in d s, \sigma_{n}<\infty, S\left(v^{i} \mathbf{1}_{j}\right) \notin t+I \text { for } 1 \leq i \leq n-1 \text { and } 1 \leq j \leq k\right)
\end{aligned}
$$




$$
\begin{aligned}
& \leq(1-\beta) \mathbb{P}\left(\sigma_{n-1}<\infty, S\left(v^{i} \mathbf{1}_{j}\right) \notin t+I \text { for } 1 \leq i \leq n-1 \text { and } 1 \leq j \leq k\right) \\
& \leq \ldots \leq(1-\beta)^{n}
\end{aligned}
$$

for all $n \geq 1$, where $\mathbf{1}_{j}:=1 \ldots 1$ ( $j$ times). Consequently, if $\Pi(I)=\infty$, then

$$
E_{i}:=\left\{\sigma_{i}<\infty \text { and } S\left(v^{i} \mathbf{1}_{j}\right) \in t+I \text { for some } 1 \leq j \leq k\right\}
$$

occurs a.s. for at least one $i \geq 1$, that is, $\nu_{1}:=\inf \left\{i \geq 1: E_{i}\right.$ occurs $\}<\infty$ a.s. on $\{\Pi(I)=\infty\}$. But the previous argument can be repeated (using again the strong Markov property) for the sequence $E_{\nu_{1}+1}, E_{\nu_{1}+2}, \ldots$ to infer $\nu_{2}:=\inf \left\{i>\nu_{1}: E_{i}\right.$ occurs $\}<\infty$ a.s. on $\{\Pi(I)=\infty\}$ and thus via induction that indeed $\{\Pi(I)=\infty\}=\limsup _{i \rightarrow \infty} E_{i}$ a.s. We have thus shown that

$$
\{\Pi(I)=\infty\}=\limsup _{i \rightarrow \infty} E_{i} \subset\{\Pi(t+I)=\infty\} \quad \text { a.s. }
$$

and thereby $H_{I}(t) \leq H_{I}(0)<1$. By interchanging the roles of $I$ and $t+I$ (now possible as $H_{t+I}(0)=$ $H_{I}(t)<1$ ), we get the reverse inequality and thus the constancy of $H_{I}$.

Case 2. $I$ unbounded.

Then $I$ equals either $\mathbb{R}$, in which case there is nothing to prove, or $(-\infty, x)$, or $(x, \infty)$ for some $x \in \mathbb{R}$. But for the last two alternatives, an even simpler geometric trials arguments than above may be employed to give the asserted result. Further details are therefore omitted.

Proof of Proposition 2.2 Let $f(s):=\sum_{j \geq 0} p_{j} s^{j}$ denote the generating function of $Z_{1}$ and observe that $p_{0}=0$ and $p_{1}<1$ ensure $f(s) \leq s$ for all $s \in[0,1]$ with equality holding iff $s \in\{0,1\}$. Put

$$
[\Pi]_{v}:=\sum_{w \in \mathbb{V}} L_{v}(w) \delta_{S(v w)-S(v)}
$$

with $L_{v}(w):=\prod_{j=1}^{k} T_{w_{j}}\left(v w_{1} \ldots w_{j-1}\right)$ for any $w=w_{1} \ldots w_{k} \in \mathbb{V}$, thus $L_{v}(w)=\frac{L(v w)}{L(v)}$ if $L(v)>0$. Then the $[\Pi]_{v}$ are just copies of $\Pi$, obtained by looking at the subtree rooted at $v$ with weights $(T(v w) \otimes$ $X(v w))_{w \in \mathbb{V}}$. Our independence assumptions further ensure that the $[\Pi]_{v}$ for $v \in \mathbb{N}^{n}$ are independent.

For any nonempty interval open $I$, note the obvious identity

$$
\Pi(t+I)=\delta_{0}(t+I)+\sum_{j=1}^{Z_{1}}[\Pi]_{j}(t-S(j)+I), \quad t \in \mathbb{R} .
$$

By combining this with the constancy of $H_{I}$ (Lemma 4.1), it follows that

$$
\begin{aligned}
H_{I}(t) & =\mathbb{P}\left([\Pi]_{j}\left(t-S(j)+I<\infty, 1 \leq j \leq Z_{1}\right)\right. \\
& =\sum_{n \geq 1} \mathbb{P}\left(Z_{1}=n\right) \mathbb{E}\left(\prod_{j=1}^{n} H_{I}(t-S(j))\right) \\
& =\sum_{n \geq 1} \mathbb{P}\left(Z_{1}=n\right) H_{I}(t)^{n} \\
& =f\left(H_{I}(t)\right)
\end{aligned}
$$


for all $t \in \mathbb{R}$, that is,

$$
H_{I}=f \circ H_{I} .
$$

But this shows $H_{I} \equiv 0$ or $\equiv 1$, for $\{s: f(s) \geq s\}=\{0,1\}$.

If $I=(-\varepsilon, \varepsilon)$ for any $\varepsilon>0$, we must still verify that $H_{\eta}=H_{\varepsilon}$ for each $\eta \in(0, \varepsilon)$. If $H_{\varepsilon} \equiv 1$, then $H_{\eta} \equiv 1$ for $\eta \in(0, \varepsilon)$ is indeed a trivial consequence of $H_{\eta} \geq H_{\varepsilon}$. If $H_{\varepsilon} \equiv 0$, then $1-H_{\varepsilon}(0)=$ $\mathbb{P}(\Pi((-\varepsilon, \varepsilon))=\infty)=1$ which in turn implies $\left.1-H_{\eta}(t)=\mathbb{P}(\Pi(t-\eta, t+\eta))=\infty\right)>0$ for some $t \in \mathbb{G}_{d}$ and thus excludes $H_{\eta} \equiv 1$. Hence, $H_{\eta} \equiv 0$ by another appeal to Lemma 4.1 and the proof of the asserted dichotomy is complete.

We proceed with two lemmata relevant to the proof of Theorem 2.4. The first provides a link between the behavior of $H(t)=\Pi((-\infty, t))$ and the following Galton-Watson process generated by ladder lines. Let $\left(\sigma_{n}\right)_{n \geq 0}$ be the possibly terminating renewal sequence of strictly descending ladder epochs associated with $\left(\bar{S}_{n}\right)_{n \geq 0}$, defined by $\sigma_{0}:=0$ and

$$
\sigma_{n}:=\inf \left\{k>\sigma_{n-1}: \bar{S}_{k}<\bar{S}_{\sigma_{n-1}}\right\}, \quad n \geq 1,
$$

where as usual $\inf \emptyset:=\infty$. Denote by $\mathcal{S}_{n}$ the HSL associated with $\sigma_{n}$, called ladder line, and observe that $\left(Z_{S_{n}}\right)_{n \geq 0}$ forms a Galton-Watson process (generated by these lines), possibly in the generalized sense that individuals have an infinite number of offspring with positive probability. If so the process is trivially supercritical.

Lemma 4.2 In the situation of Proposition 2.2 the following statements are equivalent:

(i) $H \equiv 0$, i.e., $\Pi((-\infty, x))=\infty$ a.s. for all $x \in \mathbb{R}$.

(ii) $\left(Z_{\mathcal{S}_{n}}\right)_{n \geq 0}$ is supercritical.

Proof: If (i) holds true then $\mathcal{S}_{n}$ is a.s. nonempty and thus $\left(Z_{\mathcal{S}_{n}}\right)_{n \geq 0}$ an a.s. nonextinctive supercritical Galton-Watson process. Conversely, the event of nonextinction of $\left(Z_{S_{n}}\right)_{n \geq 0}$ has positive probability under (ii), and since $S(v)<0$ for infinitely many $v \in \mathbb{T}$ on this event, we infer $\Pi((-\infty, 0))=\infty$ with positive probability which in turn implies (i) by an appeal to Proposition 2.2 .

Our second lemma is obtained by a geometric trials argument similar to the one in the proof of Proposition 2.2

Lemma 4.3 In the situation of Proposition 2.2 suppose additionally that $Q$ has finite positive mean. Then the following statements are equivalent:

(i) $H \equiv 0$.

(ii) $H_{\varepsilon} \equiv 0$ for all $\varepsilon>0$.

Proof: Clearly, it suffices to show that (i) implies (ii), that is, if any interval $(-\infty, x)$ is a.s. visited infinitely often, then we have the same for any bounded open interval $I$.

The following argument is given for the nonarithmetic case, but the modifications in the case $d=1$ are straightforward and thus omitted. For $b \geq 0$, let $\tau(b):=\inf \left\{n \geq 1: \bar{S}_{n}>b\right\}$ and $R_{b}=\bar{S}_{\tau(b)}-b$ the 
associated overshoot (the first strictly ascending ladder height for $b=0$ ). As $Q$ has finite mean, the same holds true for $R_{0}$ and $R_{b}$ converges in distribution to $R_{\infty}$, say, with distribution function

$$
\mathbb{P}\left(R_{\infty} \leq r\right)=\frac{1}{\mathbb{E} R_{0}} \int_{0}^{r} \mathbb{P}\left(R_{0}>x\right) d x, \quad r \geq 0 .
$$

Consequently, we can pick some positive $t$ and $\varepsilon$ such that

$$
p:=\inf _{b \geq t} \mathbb{P}\left(R_{b} \leq \varepsilon\right)>0 .
$$

After these observations the geometric trials argument goes as follows. Assuming (i) and thus a fortiori $\Pi((-\infty,-t))=\infty$ a.s., we can pick a (random) sequence of nodes $v^{1}, v^{2}, \ldots$ such that $S\left(v^{j}\right) \leq-t$ for each $j \geq 1$. Start with $\hat{v}^{1}:=v^{1}$ and follow the path $v^{1}, v^{1} 1, v^{1} 11, \ldots$ until the first $v^{1} \mathbf{1}_{\nu}$ with $S\left(v^{1} \mathbf{1}_{\nu}\right)>0$ or, equivalently, $S\left(v^{1} \mathbf{1}_{\nu}\right)-S\left(v^{1}\right)>-S\left(v^{1}\right)$, where $\mathbf{1}_{n}:=1 \ldots 1$ ( $n$-times) should be recalled. But our assumptions ensure that $\left(S\left(v^{1} \mathbf{1}_{n}\right)-S\left(v^{1}\right)\right)_{n \geq 1}$ is independent of $S\left(v^{1}\right)$ and having the same distribution as $\left(\bar{S}_{n}\right)_{n \geq 1}$. Consequently, by our choice of $t, \varepsilon$ and the stopping time $\nu$, there is a chance of at least $p$ that $S\left(v^{1} \mathbf{1}_{\nu}\right) \in(0, \varepsilon]$. Now pick the first node $\hat{v}^{2}$ from $v^{2}, v^{3}, \ldots$ of length $>\left|v^{1}\right|+\nu$, follow the path $\hat{v}^{2}, \hat{v}^{2} 1, \hat{v}^{2} 11, \ldots$ until $S\left(\hat{v}^{2} \mathbf{1}_{k}\right)>0$ for the first time. Again, the interval $(0, \varepsilon]$ is hit with probability at least $p$ and independent of the first trial, by the strong Markov property. Continuing this way, the interval $(0, \varepsilon]$ is hit once after an a.s. finite number of rounds and then indeed infinitely often, thus showing $\Pi((0, \varepsilon))=\infty$ a.s. But Proposition 2.2 now ensures $\Pi((x-\eta, x+\eta))=\infty$ a.s. for all $x \in \mathbb{R}$ and $\eta>0$, i.e., (ii) holds true.

Proof of Theorem 2.5: (a) We give first an argument which does not require the previous two lemmata but works only in the 1-arithmetic case. Let $\sigma=\inf \left\{n \geq 1: \bar{S}_{n}=0\right\}$ and $\mathcal{S}$ be the associated HSL. Then $Z_{\mathcal{S}}=Z_{\mathcal{S}}^{\theta}$ for all $\theta$ together with 7 in Lemma 3.2 shows that

$$
\mathbb{E} Z_{\mathcal{S}}=\mathbb{E} Z_{\mathcal{S}}^{\theta}=\mathbb{E}_{\theta}(m \Psi(\theta))^{\sigma} \mathbf{1}_{\{\sigma<\infty\}}
$$

for each $\theta \in \mathbb{D}_{\Psi}$. Now choose $\theta=\vartheta$ and notice that $\left(\bar{S}_{n}\right)_{n \geq 0}$ has drift $\Psi^{\prime}(\vartheta)=0$ under $\mathbb{P}_{\vartheta}$ and is therefore recurrent on $\mathbb{Z}$, i.e., $\mathbb{P}_{\vartheta}(\sigma<\infty)=1$. Consequently,

$$
\mathbb{E} Z_{\mathcal{S}}=\mathbb{E}_{\vartheta}(m \Psi(\vartheta))^{\sigma} \begin{cases}\leq 1, & \text { if } m \Psi(\vartheta) \leq 1 \\ >1, & \text { if } m \Psi(\vartheta)>1\end{cases}
$$

Considering once again the Galton-Watson process $\left(\hat{Z}_{n}\right)_{n \geq 0}$, say, of all particles visiting 0 with first generation size $Z_{\mathcal{S}}$, we thus infer this process be critical or subcritical, if $m \Psi(\vartheta) \leq 1$, and supercritical otherwise. In the latter case, it has a positive chance of survival, that is, $\mathbb{P}(\Pi(\{0\})<\infty)<1$. Proposition 2.2 then ensures that this probability must be 0 as claimed, in other words, the BRW is recurrent. If $m \Psi(\vartheta) \leq 1$, then almost certain extinction of $\left(\hat{Z}_{n}\right)_{n \geq 0}$ naturally gives $\mathbb{P}(\Pi(\{0\})<\infty)=1$ and hence the transience of $\left(\Pi_{n}\right)_{n \geq 0}$. In the critical case we should mention that $\mathbb{P}\left(Z_{\mathcal{S}}=1\right)=1$ is easily excluded.

(b) Suppose now we are in the nonarithmetic case. The following argument embarks on Lemma 4.3 by which it suffices to consider the function $H$ so as to assess recurrence or transience of the given BRW. By Lemma 4.2 and in the notation from there, this can be done by computing the mean offspring $\mathbb{E} Z_{\mathcal{S}_{1}}$ of the 
Galton-Watson process $\left(Z_{\mathcal{S}_{n}}\right)_{n \geq 0}$. Suppose first $m \Psi(\vartheta) \leq 1$ and notice that $S(v)<0$ for all $v \in \mathcal{S}_{1}$ in combination with $\vartheta>0$ implies

$$
Z_{\mathcal{S}_{1}}^{\vartheta}>Z_{\mathcal{S}_{1}}
$$

Consequently, by (7) of Lemma 3.2

$$
\mathbb{E} Z_{\mathcal{S}_{1}}<\mathbb{E} Z_{\mathcal{S}_{1}}^{\vartheta}=\mathbb{E}_{\vartheta}\left(m \Psi(\vartheta)^{\sigma_{1}} \mathbf{1}_{\left\{\sigma_{1}<\infty\right\}}\right) \leq 1 .
$$

The process $\left(Z_{\mathcal{S}_{n}}\right)_{n \geq 0}$ thus being subcritical we infer, by Lemma 4.2 that $\Pi((-\infty, 0))<\infty$ a.s. and thereby transience of $\left(\Pi_{n}\right)_{n \geq 0}$ as claimed.

If $e^{\vartheta \varepsilon}=m \Psi(\vartheta)>1$ (with $\varepsilon>0$ defined by this equality), consider the stopping times $\sigma_{0}^{\varepsilon} \equiv 0$,

$$
\sigma_{n}^{\varepsilon}:=\inf \left\{k>\sigma_{n-1}^{\varepsilon}: \bar{S}_{k}-\bar{S}_{\sigma_{n-1}^{\varepsilon}} \in(-\varepsilon, 0)\right\}
$$

for $n \geq 1$, and let $\mathcal{S}_{n}^{\varepsilon}, n \geq 0$, be the associated HSL. Under $\mathbb{P}_{\vartheta}$, all $\sigma_{n}^{\varepsilon}$ are a.s. finite as $\left(\bar{S}_{n}\right)_{n \geq 0}$ has drift 0 and is therefore recurrent. By another appeal to 7 ) of Lemma 3.2 .

$$
\mathbb{E} Z_{\mathcal{S}_{1}^{\varepsilon}}^{\vartheta}=\mathbb{E}_{\vartheta}(m \Psi(\vartheta))^{\sigma_{1}^{\varepsilon}} \geq m \Psi(\vartheta)
$$

which in combination with the inequality

$$
e^{-\vartheta \varepsilon} Z_{\mathcal{S}_{1}^{\varepsilon}}^{\vartheta}=\sum_{v \in \mathcal{S}_{1}^{\varepsilon}} L(v) e^{-\vartheta(S(v)+\varepsilon)}<Z_{\mathcal{S}_{1}^{\varepsilon}}
$$

leads to

$$
\mathbb{E} Z_{\mathcal{S}_{1}^{\varepsilon}}>e^{-\vartheta \varepsilon} \mathbb{E} Z_{\mathcal{S}_{1}^{\varepsilon}}^{\vartheta} \geq e^{-\vartheta \varepsilon} m \Psi(\vartheta)=1 .
$$

We thus arrive at the conclusion that the Galton-Watson process $\left(Z_{\mathcal{S}_{n}^{\varepsilon}}\right)_{n \geq 0}$ is supercritical and therefore surviving with positive probability. As

$$
\left\{Z_{\mathcal{S}_{n}^{\varepsilon}} \text { survives }\right\} \subset\left\{Z_{\mathcal{S}_{n}} \text { survives }\right\},
$$

the process $\left(Z_{\mathcal{S}_{n}}\right)_{n \geq 0}$ is also supercritical and therefore $\Pi((-\infty, 0))=\infty$ a.s. by Lemma 4.2 . This proves the recurrence of $\left(\Pi_{n}\right)_{n \geq 0}$.

\section{Extremal particle positions in a critical BRW}

Once knowing that the critical BRW is transient and thus drifting to $\infty$, it is natural ask for its minimal speed or, equivalently, the asymptotic behavior of the leftmost particle in the cloud as time goes to infinity. Define

$$
\operatorname{Min}_{n}:=\min _{|v|=n} S(v) \quad \text { and } \quad \operatorname{Max}_{n}:=\max _{|v|=n} S(v) .
$$

In the critical case, it is not surprising and in fact following from an old more general result by Biggins (1976, 1977) that

$$
\frac{\operatorname{Min}_{n}}{n} \rightarrow 0 \text { a.s. }
$$


However, by drawing on recent work of Hu and Shi (2008) (see also Alsmeyer (2007)), we can provide far more precise information about the behavior of $\operatorname{Min}_{n}$ under some additional conditions, and also about the naturally related one on the behavior of the rightmost particle (maximal speed) in the cloud. Towards this end, we make the additional assumptions hereafter that the step size distribution $Q$ has bounded support and that

$$
\mathbb{E} Z_{1}^{2}=\sum_{n \geq 1} n^{2} p_{n}<\infty
$$

The following result then follows directly from the more general Theorem $1.2 \mathrm{in}$ Hu and Shi](2008) (note that $\vartheta=1$ in this work).

Theorem 5.1 Let $\left(\Pi_{n}\right)_{n \geq 0}$ be a genuinely two-sided critical BRW satisfying $p_{0}=0, p_{1}<1, \mu(Q) \in$ $(0, \infty)$ and $(11$. Suppose further the step size distribution $Q$ to be bounded. Then (with $\vartheta$ defined by (3))

$$
\frac{1}{2 \vartheta}=\liminf _{n \rightarrow \infty} \frac{\operatorname{Min}_{n}}{\log n}<\limsup _{n \rightarrow \infty} \frac{\operatorname{Min}_{n}}{\log n}=\frac{3}{2 \vartheta} \quad \text { a.s. }
$$

as well as

$$
\frac{\operatorname{Min}_{n}}{\log n} \stackrel{\mathbb{P}}{\longrightarrow} \frac{3}{2 \vartheta} .
$$

The natural way for getting a similar result for the rightmost particle is to resort to the previous one after a reflection of the given BRW at a suitable line $x \mapsto \gamma x$. Put $\Phi(\mu):=\inf \left\{e^{\theta \mu} \Psi(\theta): \theta \leq 0\right\}$ which is strictly decreasing and continuous on $\{\mu: 0<\Phi(\mu)<\Psi(\vartheta)\} \subset(\mu(Q), \infty)$. Defining further

$$
\gamma:=\sup \{\mu: m \Phi(\mu) \geq 1\}
$$

Biggins (1976) also showed that

$$
\frac{\operatorname{Max}_{n}}{n} \rightarrow \gamma \text { a.s. }
$$

Now, if $\gamma$ is given along with a $\kappa>0$ such that 12 holds together with

$$
1=m \Phi(\gamma)=m e^{-\kappa \gamma} \Psi(-\kappa) \quad \text { and } \quad \gamma=-\frac{\Phi^{\prime}(-\kappa)}{\Phi(-\kappa)}
$$

(the latter identity is an equivalent statement for that the derivative of $\theta \mapsto e^{\theta \gamma} \Psi(\theta)$ at $\theta=-\kappa$ be 0 ), then one can easily check that the reflected BRW $\left(\widehat{\Pi}_{n}\right)_{n \geq 0}$, defined as

$$
\widehat{\Pi}_{n}:=\sum_{|v|=n} L(v) \delta_{\gamma n-S(v)}
$$

is again genuinely two-sided with positive drift, critical and satisfying all conditions of Theorem 5.1. As for its leftmost particle position $\widehat{\operatorname{Min}}_{n}$ at time $n$, we can thus apply Theorem 5.1 and have also the obvious relation

$$
\operatorname{Max}_{n}=\gamma n-\widehat{\operatorname{Min}}_{n}
$$

for all $n \geq 0$. Thus we finally arrive at the following result for the rightmost particle position. 
Theorem 5.2 Under the same conditions as in Theorem 5.1 let $\gamma$ be defined by (12). Suppose additionally the existence of a $\kappa>0$ such that the pair $(\gamma, \kappa)$ satisfies [13]. Then

$$
-\frac{3}{2 \kappa}=\liminf _{n \rightarrow \infty} \frac{\operatorname{Max}_{n}-\gamma n}{\log n}<\limsup _{n \rightarrow \infty} \frac{\operatorname{Max}_{n}-\gamma n}{\log n}=-\frac{1}{2 \kappa} \quad \text { a.s. }
$$

as well as

$$
\frac{\operatorname{Max}_{n}-\gamma n}{\log n} \stackrel{\mathbb{P}}{\longrightarrow}-\frac{3}{2 \kappa}
$$

\section{References}

G. Alsmeyer. Minimal position and critical martingale convergence in branching random walks. Technical report, Universität Münster, 2007.

I. Benjamini and Y. Peres. Markov chains indexed by trees. The Annals of Probability, 22(1):219-243, 1994. ISSN 00911798.

J. D. Biggins. The first- and last-birth problems for a multitype age-dependent branching process. $A d$ vances in Appl. Probability, 8(3):446-459, 1976. ISSN 0001-8678.

J. D. Biggins. Chernoff's theorem in the branching random walk. J. Appl. Probability, 14(3):630-636, 1977. ISSN 0021-9002.

J. D. Biggins and A. E. Kyprianou. Seneta-heyde norming in the branching random walk. The Annals of Probability, 25(1):337-360, 1997. ISSN 00911798.

J. D. Biggins and A. E. Kyprianou. Measure change in multitype branching. Advances in Applied Probability, 36(2):544-581, 2004. ISSN 00018678.

J. D. Biggins and A. E. Kyprianou. Fixed points of the smoothing transform: the boundary case. Electron. J. Probab., 10:no. 17, 609-631 (electronic), 2005. ISSN 1083-6489.

N. H. Bingham and R. A. Doney. Asymptotic properties of super-critical branching processes ii: Crumpmode and jirina processes. Advances in Applied Probability, 7(1):66-82, 1975. ISSN 00018678.

B. Chauvin. Product martingales and stopping lines for branching brownian motion. The Annals of Probability, 19(3):1195-1205, 1991. ISSN 00911798.

F. Comets, M. V. Menshikov, and S. Y. Popov. One-dimensional branching random walk in a random environment: a classification. Markov Process. Related Fields, 4(4):465-477, 1998. ISSN 1024-2953. I Brazilian School in Probability (Rio de Janeiro, 1997).

R. Durrett and T. M. Liggett. Fixed points of the smoothing transformation. Z. Wahrsch. Verw. Gebiete, 64(3):275-301, 1983. ISSN 0044-3719.

N. Gantert and S. Müller. The critical branching Markov chain is transient. Markov Process. Related Fields, 12(4):805-814, 2006. ISSN 1024-2953. 
Y. Hu and Z. Shi. Minimal position and critical martingale convergence in branching random walks, and directed polymers on disordered trees. arXiv:math/0702799v3, Mar. 2008.

P. Jagers. General branching processes as Markov fields. Stochastic Process. Appl., 32(2):183-212, 1989. ISSN 0304-4149.

A. E. Kyprianou. Martingale convergence and the stopped branching random walk. Probab. Theory Related Fields, 116(3):405-419, 2000. ISSN 0178-8051.

F. P. Machado and S. Y. Popov. One-dimensional branching random walks in a Markovian random environment. J. Appl. Probab., 37(4):1157-1163, 2000. ISSN 0021-9002.

F. P. Machado and S. Y. Popov. Branching random walk in random environment on trees. Stochastic Process. Appl., 106(1):95-106, 2003. ISSN 0304-4149.

F. P. Machado, M. V. Menshikov, and S. Y. Popov. Recurrence and transience of multitype branching random walks. Stochastic Process. Appl., 91(1):21-37, 2001. ISSN 0304-4149.

M. V. Menshikov and S. E. Volkov. Branching Markov chains: qualitative characteristics. Markov Process. Related Fields, 3(2):225-241, 1997. ISSN 1024-2953. 
